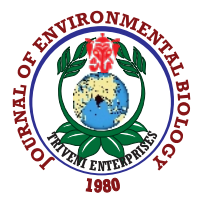

\title{
Optimization of nutritive factors in culture medium for the growth of hairy root and ajmalicine content in sarpgandha, Rauwolfia serpentina
}

\author{
P. Bhagat', S.K. Verma', S. Yadav', A.K. Singh ${ }^{2}$, G.K. Aseri' and N. Khare ${ }^{1 *}$ \\ ${ }^{1}$ Amity Institute of Microbial Technology, Amity University, Jaipur - 302 015, India \\ ${ }^{2}$ Division of Genomic Resources, National Bureau of Plant Genetic Resources, New Delhi - 110 012, India \\ *Corresponding Author Email : neerajsnkhare@gmail.com
}

\begin{tabular}{lll}
\hline Paper received: 31.10 .2019 & Revised received: 08.01.2020 Accepted:01.04.2020 \\
\hline
\end{tabular}

\section{Abstract}

Aim: To optimize nutritive factors for hairy root development and ajmalicine content in Rauwolfia serpentina.

Methodology : Optimization of nutritive factor was performed with 150 $\mathrm{mg}$ hairy root in $50 \mathrm{ml}$ culture medium.Different ratio of nitrate and ammonium ions $(0: 20,10: 20,20: 20,40: 20,60: 20,20: 0,20: 10,20: 40$, 20:60), different phosphate concentration $(0.625-5.0 \mathrm{mM})$, different culture medium (MS, B5, $1 / 2$ MS, $1 / 2 \mathrm{~B} 5$ ), different $\mathrm{pH}(4.0-7.0)$, different concentration ( $2 \%-4 \%$ ) of carbon sources (sucrose, fructose, glucose, galactose) were evaluated for dry weight (growth index) and ajmalicine content enhancement.

Results : Growth of hairy root was observed best in $1 / 2$ MS media. Sucrose was found significantly best among the other tested carbon sources in terms of higher growth index (4.31)and ajmalicine content $(0.0068 \mathrm{mg} \mathrm{g}$ ${ }^{1}$ d.wt.) at $3 \%$ and $3.5 \%$ sucrose concentration. $\mathrm{NH}_{4}{ }^{+}$to $\mathrm{NO}_{3}{ }_{3}^{-}$ratio $(\mathrm{mM})$ of 40:20 and 20:10 was significantly optimum for growth index (7.12) and ajmalicine content $\left(0.0112 \mathrm{mg} \mathrm{g}^{-1} \mathrm{~d}\right.$. wt.). Culture media supplemented with $0.625 \mathrm{mM}$ phosphate concentration resulted into significantly higher ajmalicine content $\left(0.0074 \mathrm{mg} \mathrm{g}^{-1} \mathrm{~d}\right.$. wt.). However,increase in phosphate concentration $(0.625-5.0 \mathrm{mM})$ resulted into continuous enhancement of growth index. Optimum pH of 6.0 and 6.5 was observed suitable for higher growth index (5.19) and ajmalicine content (0.0076 $\mathrm{mg} \mathrm{g}^{-1} \mathrm{~d} . w \mathrm{t}$.), respectively.

Interpretation : Standardization of nutritive factor in culture media resulted in a development of alternative strategy for large scale production of ajmalicine, a pharmaceutically important alkaloid.

Keywords : Ajmalicine, Growth index, Hairy root culture, Rauwolfia serpentina

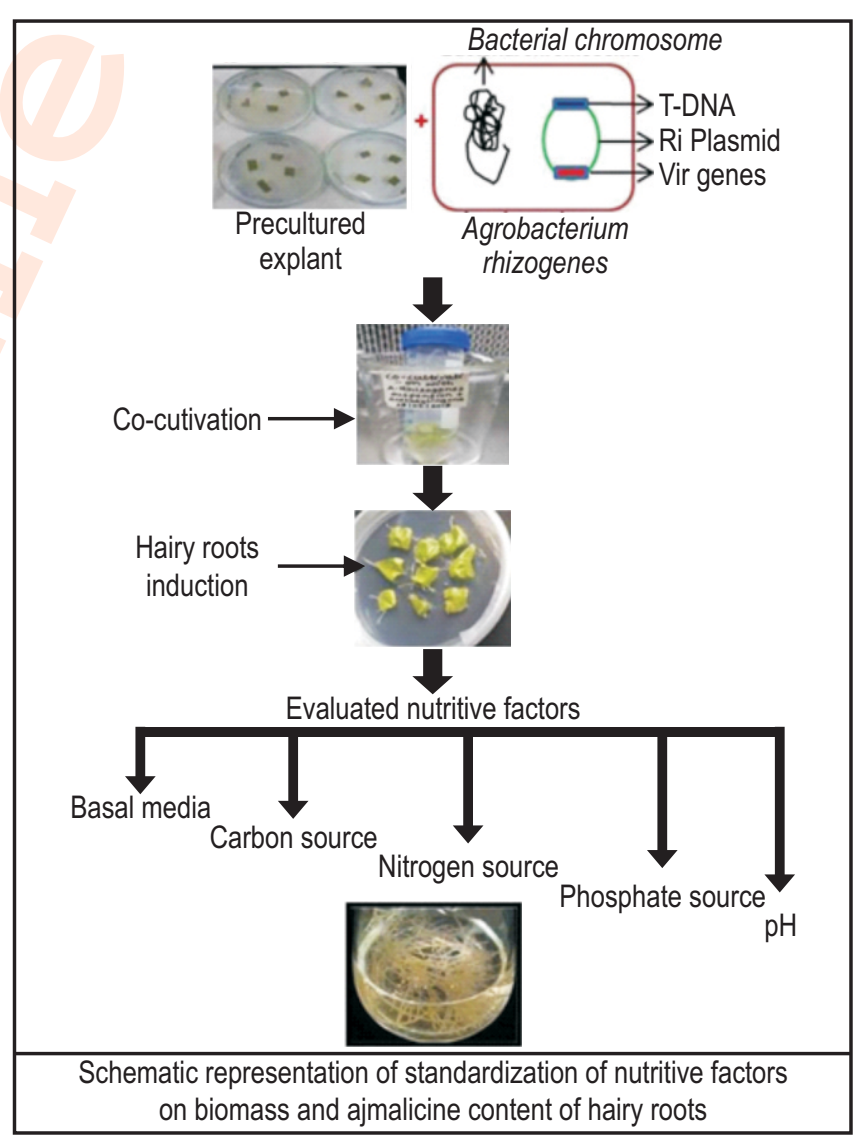

How to cite : Bhagat, P., S.K. Verma, S. Yadav, A.K. Singh, G.K. Aseri and N. Khare: Optimization of nutritive factors in culture medium for the growth of hairy root and ajmalicine content in sarpgandha, Rauwolfia serpentina. J. Environ. Biol., 41, 1018-1025 (2020). 


\section{Introduction}

Rauwolfia serpentina, popularly known as sarpgandha is a perennial shrub of family Apocynaceae. This plant is a hub of more than 100 medicinally important terpenoidindole alkaloid mainly reserpine, ajmalicine, ajmaline, yohimbine etc. Majority $(85-90 \%)$ of these alkaloids are found mainly in roots (Pathania, 2013). Ajmalicine is one of the principle alkaloid known for the treatment of hypertension. $R$. serpentina is grouped under the category of endangered plant by International Union for the Conservation of Nature and Natural Resources due to its overexploitation for medicinal purposes (Shetty et al., 2014). Organic synthesis of these alkaloids is expensive and tedious process due to its complex structure with regio- and stereo-specific actions (Pathania, 2013). Various plant tissue culture strategies have been employed as an alternative to produce these alkaloids viz. cell suspension culture, in-vitro clonal propagation etc., but has limited application due to the concern of genetic and biosynthetic variability of cell culture (Liu et al., 2012). However, hairy root culture of $R$. serpentina has substantiated its utility among all plant tissue culture techniques due to its biosynthetic steadiness, consistency in prolonged and economic production of alkaloids (Guillon et al., 2006; Häkkinen et al., 2016). Currently, various commercially important secondary metabolites have been produced by hairy root cultures (Lorence et al., 2004; Chang et al., 2005). Hairy roots have specific nutrient requirements, hence the optimization of culture condition is essentially required for every genotype (Sivakumar et al., 2005; Gai et al., 2015). Influence of carbon source on different the production of secondary metabolites has been reported since long back (Mantell and Smith, 1983). The concentration and ration of nitrogen sources $\left(\mathrm{NH}_{4}^{+} / \mathrm{NO}_{3}{ }^{-}\right)$is a decisive factor of culture media crucial for cell development and proliferation, root growth cell morphology regeneration potential and secondary metabolite accumulation (Srivastava and Srivastava, 2007; Zhang et al., 2007; Cui et al., 2010; Khare et al., 2010; Sivakumar et al., 2005). Standardization of culture conditions for $A$. rhizogenes mediated hairy root development from suitable explant of $R$. serpentina has been reported (Benjamin et al., 1993; Sarma et al., 1997; Mehrotra et al., 2013; Ray et al., 2014), however, studies on optimization of nutritive factors for the augmentation of secondary metabolites is meagre. Therefore, the present study was carried out to examine the nutrient factors and $\mathrm{pH}$ for the growth of hairy root and enhanced production of ajmalicine, a pharmaceutically important secondary metabolite from a perennial shrub, Rauwolfia serpentina.

\section{Materials and Methods}

Plant material and its transformation: Plants of Rauwolfia serpentina were procured from Herbal Garden, Rishikesh, Uttarakhand, India. Young leaves were used as explants for transformation experiments. Agrobacterium rhizogenes(strain MTCC 532) was procured from the Institute of Microbial
Technology, Chandigarh, India. MTCC 532 was grown in nutrient broth at $26^{\circ} \mathrm{C}$ under shaking condition (200 rpm). Optical density $\left(\mathrm{OD}_{600}\right)$ of bacterial cultures was recorded at $600 \mathrm{~nm}$. Explants were surface sterilized with $0.1 \% \mathrm{HgCl}_{2}$ solution and $70 \%$ ethanol. Surface sterilized explants were cultured (Pre-culture period) on MS medium for 2 days. Bacterial culture was grown upto desired $\mathrm{OD}_{600}$, centrifuged at $6000 \mathrm{rpm}$ for $5 \mathrm{~min}$ and pellet dissolved in liquid MS medium. Pre-cultured leaves were infected with bacterial suspension with gentle shaking, blot dried and cultured on MS medium for 2 days (co-cultivation period). Co-cultivated explants were washed with $250 \mathrm{mg} \mathrm{l}^{-1}$ cefotaxime, blot dried and cultured on selection medium ( $1 / 2$ MS medium with $250 \mathrm{mg} \mathrm{l}^{-1}$ cefotaxime) for hairy root initiation (Khare et al., 2010). Explants which were not infected with Agrobacterium were utilized as control.

Molecular analysis of transgenic roots: Genomic DNA from normal roots (Non-transformed) and transgenic hairy roots were isolated using Plant DNeasy mini kit following the manufacturer's manual (Qiagen). Plasmid DNA was isolated from A. rhizogenes using plasmid isolation kit (Qiagen), following the manual and was utilized as positive control. PCR was performed for identifying the presence of $360 \mathrm{bp}$ of the rolA gene using gene specific primers set: forward primer 5'GGAATTAGCCGGACT AAACG-3' and reverse 5'-CCGGCGTGGAAATGAATCG-3'.

Proliferation of hairy roots : Hairy roots were induced from the explants within 20 days to selection medium transfer. Putative transgenic hairy roots were excised from the explants and cultured on $1 / 2 \mathrm{MS}$ solid medium containing $3 \%$ sucrose at $26^{\circ} \mathrm{C}$ under dark condition and sub-cultured every 3 week. Five lines were randomly selected among the PCR positive hairy roots lines and $150 \mathrm{mg}$ of each lines were cultured in $1 / 2 \mathrm{MS}$ liquid medium (50 $\mathrm{ml}$ ) with $3 \%$ sucrose in an incubator shaker $(200 \mathrm{rpm})$ at $26^{\circ} \mathrm{C}$ under dark condition for optimization of nutritive factors.

Quantitation of ajmalicine through HPLC : Quantitative estimation of ajmalicine in transgenic and control hairy roots was performed by reverse-phase high-performance liquid chromatography (HPLC). HPLC was performed with $20 \mu \mathrm{l}$ of sample, $1 \mathrm{ml} \mathrm{min}^{-1}$ flow rate and detection at $298 \mathrm{~nm}$. HPLC consisted of C18 Column and photodiode array detector (Shimadzu, Kyoto, Japan). The samples were crushed with liquid nitrogen into powdered form and extracted with methanol. Chromatographic elution was performed in methanol and diammonium hydrogen orthophosphate in water (70:30) (Satdive et al., 2003). Samples (crude extract) were analyzed in triplicates.

Optimization of nutritive factors: Optimization of nutritive factor was done on the basis of growth index and ajmalicine content. Growth index (GI) was calculated according to the given below formula:

$$
\mathrm{GI}=\frac{\text { harvested dry weight }- \text { inoculated dry weight }}{\text { inoculated dry weight }}
$$


MS medium (Murashige and Skoog, 1962), 1/2 MS, B5 medium (Gamborg et al., 1968) and $1 / 2 \mathrm{~B} 5$ culture medium were evaluated for selection of suitable culture medium. Data were recorded after 2, 4, 6, 8, 10 and 12 weeks. Suitable carbon source was selected among the glucose, galactose, sucrose and fructose within the concentration range of $2 \%$ - $4 \%$ after standardization of culture medium. Optimization of nitrogen content was performed by supplementing different concentration and ratio of ammonia $\left(\mathrm{NH}_{4}^{+}\right)$and nitrate $\left(\mathrm{NO}_{3}\right)$ in $1 / 2 \mathrm{MS}$ culture medium. The evaluated ratios of $\mathrm{NH}_{4}^{+}$to $\mathrm{NO}_{3}$ were $0: 20,10: 20$, $20: 20,40: 20,60: 20,20: 10,20: 40,20: 60$ and 20:0 mM. Optimization of phosphate levels included supplementation of $\mathrm{KH}_{2} \mathrm{PO}_{4}$ at various concentrations $(0.625-5 \mathrm{mM})$ in $1 / 2 \mathrm{MS}$ culture medium. The $\mathrm{pH}$ of culture medium was also optimized within the range of $4.0-7.0$. Data of growth index and ajmalicine content were recorded after 30 days.

Statistical analyses : All the parameters were evaluated in five independent replicates. Data were examined for significant differences using IBM SPSS Statistics 20 program. Different letters indicate significant difference (PÂ0.05).

\section{Results and Discussion}

Leaf explants were pre-cultured for 2 days and cocultivated with $A$. rhizogenes $\left(\mathrm{OD}_{600} 0.6\right)$ for $15 \mathrm{~min}$ for its attachment to explants. After co-cultivation of 2 days, the infected explants were washed with $250 \mathrm{ppm}$ cefotaxime and cultured on selection medium for inducing hairy root. Transgenic hairy roots were emerged from the explants within 18 days after transferring on to selection medium (Fig. 1A). No hairy roots emerged from the control explants. Hairy roots $(150 \mathrm{mg})$ from 5 randomly selected PCR positive lines were transferred to liquid medium for optimization of nutritive factors (Fig. 1B). All the putative hairy root lines were confirmed through amplification of rolA gene. $360 \mathrm{bp}$ amplified product was observed on $1.2 \%$ agarose gel using gene specific primes (Fig. 1C).
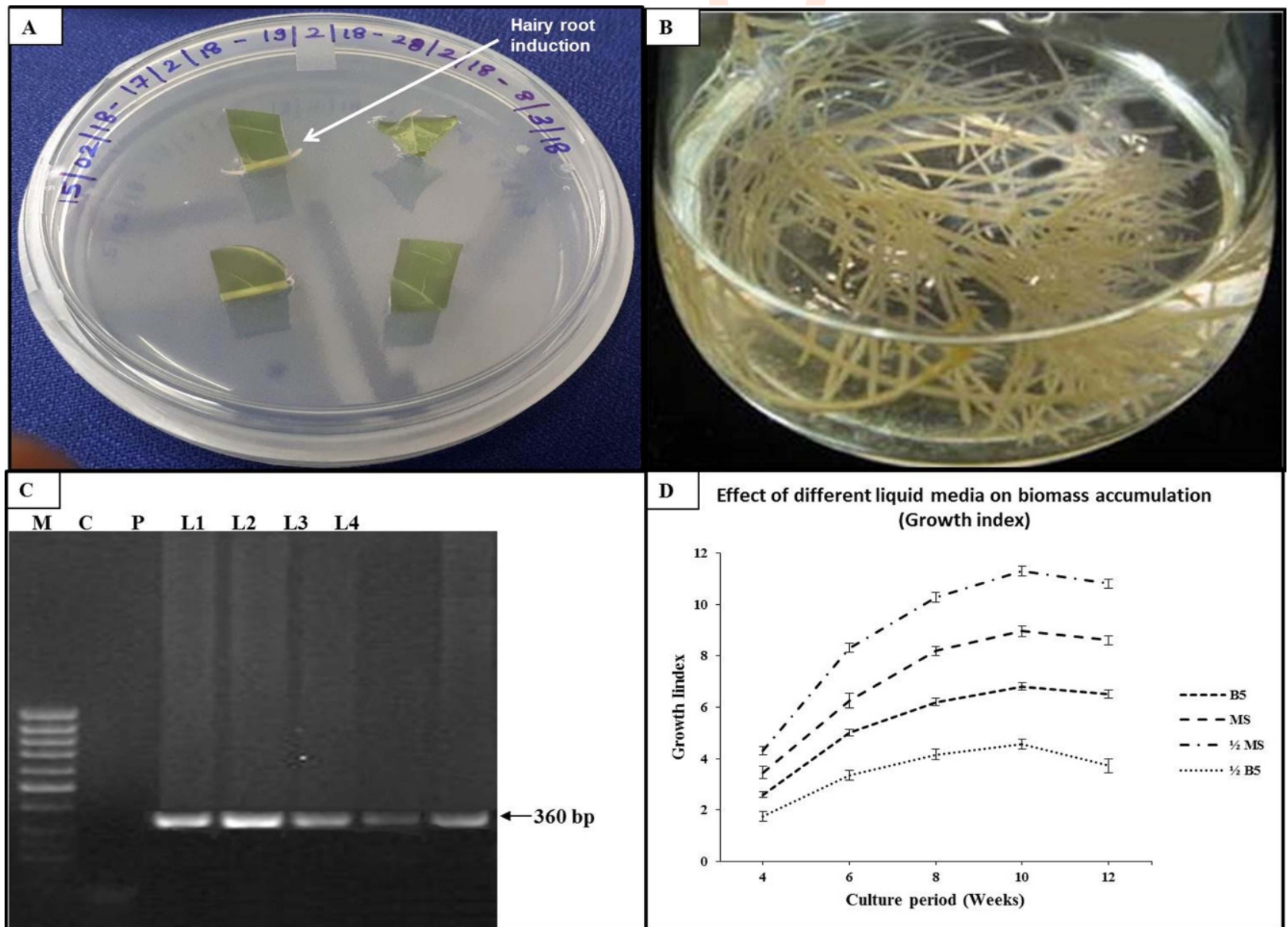

Fig. 1: A. rhizogenes mediated hairy root induction from leaf explants of $R$. serpentina (A) Induction of hairy roots form leaf explants with $A$. rhizogenes; (B) Proliferation of hairy roots from $150 \mathrm{mg}$ hairy roots on liquid $1 / 2 \mathrm{MS}$ medium; (C) PCR analysis of putative hairy roots and normal roots of $R$. serpentina plants forrol Agene. Lane 1: 100 bp DNA ladder, Lane 2: normal roots confirming the absence of rolAgene, Lane 3: DNA from plasmid as positive control, Lane 4,5,6,7: hairy roots confirming the presence of rolAgene and (D) effect of growth medium on growth index. 
$1 / 2$ MS medium was observed best among the evaluated four culture media (MS, $1 / 2$ MS, B5, $1 / 2$ B5) (Fig. 1D) in all the tested period (week 4, 6, 8,10, 12). Dry weight (growth index) consistently increased on increasing the culture period due to the enhancement of growth and number of hairy roots. Significantly higher growth index (11.31) of hairy roots was observed in $1 / 2 \mathrm{MS}$ culture media followed by MS (8.96), B5 (6.81), 1/2 B5 (4.57) at week 10 (Fig. 1D). It was also observed that hairy root biomass was higher in liquid medium as compared to solid medium (culture medium with $0.8 \%$ agar) (Data not shown) in all the tested period similar to the previous findings of Huang et al. (2014). Culture media has a critical impact on the induction and development of hairy roots, especially high salt containing medium such as MS which are well suited for the development of hairy roots in many plants such as Datura stramonium, Gossypium hirsutum L., Catharanthus roseus, With aniasomnifera etc. (Hilton et al., 1990; Giri and Narasu, 2000; Murthy et al., 2008; Verma et al., 2015; Hanafya et al. 2016). Culture medium parameters such as nutrients composition, salt strength, phosphate levels, plant growth regulators type and concentration, carbon/nitrogen ratios, combination of carbon and nitrogen sources etc., influences the signal transduction systems which is required for the expression of genes related to secondary metabolites (Sharafi et al., 2013). MS and B5 media both have almost similar concentration of calcium and phosphorous, however, nitrogen content is high in B5 medium ( $8.26 \mathrm{mM})$ as compared to the MS medium (5.22 mM). In the present study, $1 / 2$ MS was found suitable for hairy root development findings which corroborate the previous findings (Udomsuk et al., 2009; Xu et al., 2009; Długosz et al., 2018) because nitrogen concentration is lowest in $1 / 2$ MS medium. Plants requirements for nutrients is fulfilled by the roots. Roots develop profusely if low strength medium is provided. $1 / 2 \mathrm{MS}$ medium contains low nitrogen content along with low concentration of other essential nutrients. High nitrogen content inhibits root development. In $1 / 2$ MS medium, macro-element concentrations were reduced by half as compared to MS medium. Nitrogen is rapidly metabolized by plant tissues and plays a key factor required for the growth of

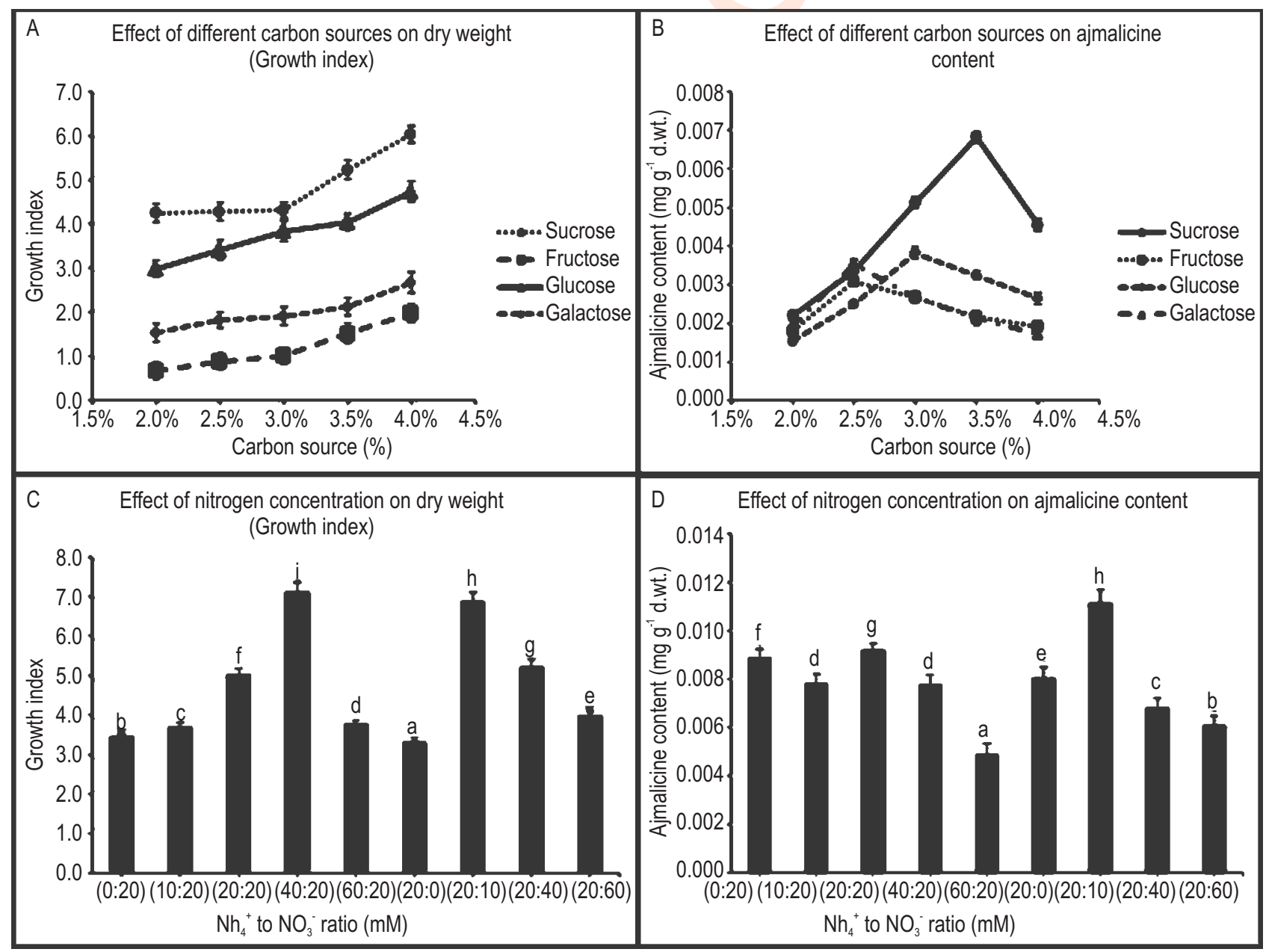

Fig. 2 : Effect of different concentration of macro elements on $R$. serpentina hairy roots after 30 days of culture; (A) carbon sources on growth index; (B) carbon sources on ajmalicine content; $(C)$ nitrogen sources on growth index and (D) nitrogen sources on ajmalicine content. 
roots. However, elevated level of nitrogen inhibited the development of hairy root (Han et al. 2001).

Plant cell culture requires sucrose as a most favourable source of carbon for its development and biosynthesis of secondary metabolite. In the present study, sucrose was also found to be superior carbon source as compared to glucose, galactose and fructose for proliferation and ajmalicine content of $R$. serpentina hairy root. Standardization of carbon source was performed by supplementing different types and concentration of carbon source in $1 / 2 \mathrm{MS}$ culture medium with $20: 40 \mathrm{NH}_{4}^{+}$to $\mathrm{NO}_{3}$ "ratio (unmodified MS). In this study, significantly elevated growth index (4.31) of hairy root was observed at $3 \%$ sucrose concentration (Fig. 2A), while on the other hand culture medium amended with $3.5 \%$ sucrose was suitable for higher ajmalicine content ( $0.0068 \mathrm{mg} \mathrm{g}^{-1}$ d.wt.) (Fig. 2B). Sugar concentration plays a critical role in biomass accumulation and profusion of lateral roots (Nguyen et al., 1992; Nin et al., 1997; Malamy and Ryan,
2001; Lourenco et al., 2002). Sucrose also elicits similar response in enhancing secondary metabolite production, and its low or high concentration severely affects the yield (Yu et al., 1996; Skala et al., 2015). Hence, the observations made in this study corroborate the previous findings of correlation of sucrose concentration with enhancement of hairy root biomass (dry weight) and ajmalicine content.

Combinations and concentration of $\mathrm{NH}_{4}^{+}$and $\mathrm{NO}_{3}{ }^{\prime \prime}$ contribute a fundamental role in root growth and secondary metabolite accumulation. Nitrogen is the most important macro element which was absorbed by the plants, both as nitrate $\left.\left(\mathrm{NO}_{3}\right)^{\prime}\right)$ and ammonium $\left(\mathrm{NH}_{4}^{+}\right)$. Standardization of nitrogen sources were carried out by supplementing of different concentration and combination of ammonia $\left(\mathrm{NH}_{4}^{+}\right)$and nitrate $\left(\mathrm{NO}_{3}\right)$ in $1 / 2 \mathrm{MS}$ culture medium with $3.5 \%$ sucrose and $1.25 \mathrm{mM}$ phosphate (unmodified MS). In the present study, significantly higher biomass (7.12) was observed at low $\mathrm{NO}_{3}{ }^{\prime}$ content $\left(40: 20 \mathrm{NH}_{4}^{+} / \mathrm{NO}_{3}{ }^{\prime}\right.$ ratio) (Fig. $2 \mathrm{C}$ ).

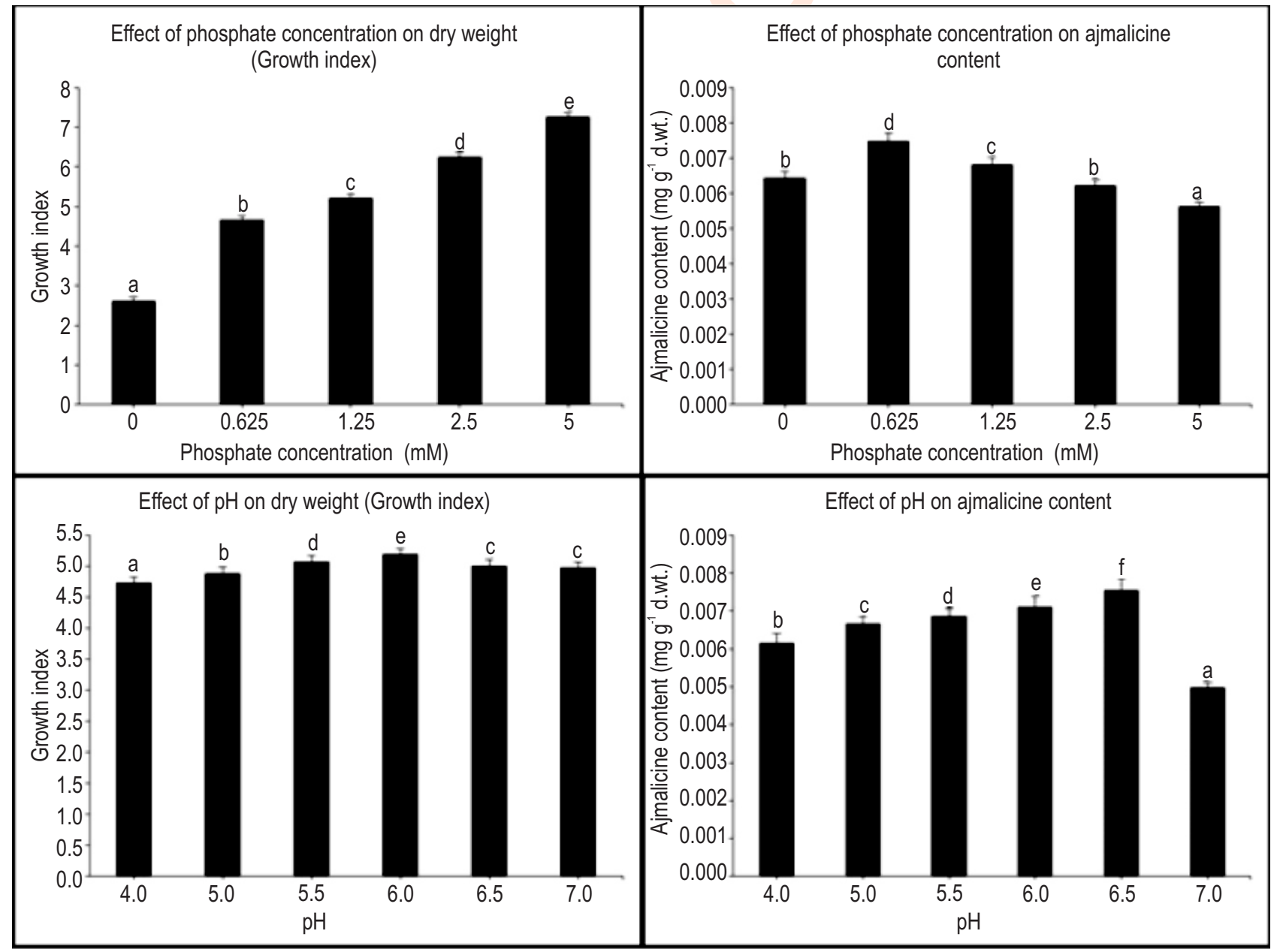

Fig. 3 : Effect of phosphate concentration and pH on R. serpentina hairy roots after 30 day of culture: (A) Phosphate concentration on growth index; (B) Phosphate concentration on ajmalicine content; (C) Different pH on growth index and (D) pH on ajmalicine content. 
Significantly higher ajmalicine content $\left(0.0112 \mathrm{mg} \mathrm{g}^{-1} \mathrm{~d} . \mathrm{wt}\right.$.) was also observed at low $\mathrm{NO}_{3}{ }_{3}$ concentration $\left(20: 10 \mathrm{NH}_{4}^{+} / \mathrm{NO}_{3}\right.$ " ratio) (Fig. 2D). Our results corroborate the previous findings that low $\mathrm{NO}_{3}$ " concentration favours elevated accumulation of secondary metabolites and very high $\mathrm{NH}_{4}^{+}$concentration negatively affects the growth due to toxicity and reduced $\mathrm{NO}_{3}$ "absorption (Jung et al., 1994; Crawford et al., 1995; Bensaddek et al., 2001; Lourenco et al., 2002; Praveen et al., 2013). Hence, in the present study, type of nitrogen source and its concentration in the medium is accountable for differential accumulation of ajmalicine and hairy rootgrowth.

The concentration of phosphate in culture media also influences the stimulation of hairy root development and secondary metabolite content in hairy roots. Standardization of phosphate source was done by supplementing carbon source in $1 / 2 \mathrm{MS}$ culture medium with $3.5 \%$ sucrose and $\mathrm{NH}_{4}^{+}$to $\mathrm{NO}_{3}{ }^{\prime}$ in 20:40 ratio (unmodified MS). In the present study, low phosphate concentration $(0.625 \mathrm{mM})$ was found optimal for significantly high ajmalicine content $\left(0.0074 \mathrm{mg} \mathrm{g}^{-1} \mathrm{~d} . w \mathrm{wt}\right.$ ) (Fig. 3A) On the other hand, growth index consistently increased on increasing the phosphate concentration within the tested concentration $(0.625-$ $5.0 \mathrm{mM}$ ) (Fig. 3B). Our results corroborate the previous findings (Dunlop et al., 1991; Taya et al.,1992; Pavlov et al., 2005) that low phosphate concentration favours the secondary metabolite content. Pavlov et al. (2005) reported that low concentration of phosphate supplemented media enhanced hairy root development and betalain content in hairy roots of red beet (Beta vulgaris). Interestingly, few reports elucidate the elevation of secondary metabolite content in the absence of phosphate in culture medium (Dunlop et al., 1991; Taya et al.,1992). It is well established fact that phosphate develops a negative control on the biosynthesis of secondary metabolites, hence, its low level is used in production medium. Phosphate deprivation triggers various biochemical cross talk signals, to increase its mobilization and reduce its utilization. Such changes in metabolism results in lower requirement for phosphate and enhancement of various secondary metabolites.

Acidic $\mathrm{pH}$ results in high hydrogen ion, aluminium ion and inhibition of cation uptake which are lethal to root system, while on the other hand at basic $\mathrm{pH}$ micronutrients are unavailable for root absorption due to bound form (Mantell and Smith, 1983; Shivakumar et al., 2005; Naik et al., 2010; Gai et al., 2015). pH is also crucial for the uptake of nitrogen which affects the assimilation of other essential micro- and macro-elements (Lang and Kaiser, 1994). Standardization of pH source was performed with different $\mathrm{pH}$ of $1 / 2 \mathrm{MS}$ culture medium with $3.5 \%$ sucrose, 1.25 $\mathrm{mM}$ phosphate, 20:40 $\mathrm{NH}_{4}^{+}$to $\mathrm{NO}_{3}$ 'ratio (unmodified MS). In this study, pH 6.0 and 6.5 was optimal for significant higher growth index (5.19) (Fig. 3C) and higher ajmalicine content $(0.0076 \mathrm{mg} \mathrm{g}$ 'd.wt.) (Fig. 3D), respectively. Growth index and ajmalicine content both were significantly reduced on further increase in $\mathrm{pH}$.
Our findings corroborate with the previous findings (Sandra et al., 1999; Young-Am et al., 2000; Sivakumar et al., 2005;) Sivakumar et al. (2005) observed that too low pH enhances the levels of aluminium and hydrogen in the culture medium of Panax ginseng, which ultimately leads to toxicity. Higher pH creates scarcity of bound form of micro nutrients essential for hairy root uptake. $\mathrm{pH}$ of 6.5 was observed best for ginsenoside production in hairy root of Panax ginseng. Significantly higher indigo production was observed in Polygonum tinctorium at pH 5.7 (Young-Am et al., 2000). Similar finding was also observed by Sandra et al. (1999) for the elevated production of scopolamine and hyoscyamine in hairy roots of Brugmansia candida.

Optimization of nutritive enhances uptake of nutrient which are crucial for the growth and ajmalicine production in hairy roots of Rauwolfia serpentina. In the present study, $1 / 2$ MS media (pH 6.5) supplemented with $3.5 \%$ sucrose, $20: 10 \mathrm{mM}$ ration of $\mathrm{NH}_{4}^{+}$to $\mathrm{NO}_{3}^{-}$and $0.625 \mathrm{mM}$ phosphate concentration was significantly observed for enhanced ajmalicine content. Hence, it can be concluded that optimized Rauwolfia serpentina hairy root culture system is a promising technology for further higher production of ajmalicine as compared to other established technology.

\section{Acknowledgments}

The authors express sincere thanks to the Science and Engineering Research Board, Department of Science and Technology, Government of India for supporting research. We also thank Amity University, Rajasthan, India for providing necessary infrastructure and facilities to carry out the research work.

\section{References}

Benjamin, B.D., G. Roja and M.R. Heble: Agrobacterium rhizogens mediated transformation of Rauwolfia serpentina: Regeneration and alkaloid synthesis. Plant Cell Tissue Organ Cult., 35, 253-257 (1993).

Bensaddek, L., F. Gillet, J.E.N. Saucedo and M.A. Fliniaux: The effect of nitrate and ammonium concentrations on growth and alkaloid accumulation of Atropa belladonna hairy roots. J. Biotechnol., 85, $35-40$ (2001).

Crawford, N.M.: Nitrate: Nutrient and signal for plant growth. Plant Cell, 7, 859 (1995)

Cui, X.H., H.N. Murthy, C.H. Wu and K.Y. Paek: Adventitious root suspension cultures of Hypericum perforatum: Effect of nitrogen source on production of biomass and secondary metabolites. In Vitro Cell Dev. Biol. Plant., 46, 437-444 (2010).

Chang, C.K., K.S. Chang, Y.C. Lin, S.Y. Liu and C.Y. Chen: Hairy root cultures of Gynostemma pentaphyllum (Thunb.) Makino: A promising approach for the production ofgypenosides as an alternative of ginseng saponins. Biotechnol. Lett., 27, 1165-1169 (2005).

Długosz, M., M. Markowski and C. Pączkowski: Source of nitrogen as a factor limiting saponin production by hairy root and suspension 
cultures of Calendula officinalis L. Acta Physiol. Plant., 40, 35 (2018).

Dunlop, D.S. and W.R. Curtis: Synergistic response of planthairy-root cultures to phosphate limitation and fungal elicitation. Biotechnol. Prog., 7, 434-438 (1991).

Gai, Q.Y., J. Jiao, M. Luo, Z.F. Wei, Y.G. Zu, W. Ma and Y.J. Fu: Establishment of hairy root cultures by Agrobacterium rhizogenes mediated transformation of Isatis tinctoria L. for the efficient production of flavonoids and evaluation of antioxidant activities. PLOS ONE, 10, e0119022 (2015).

Gamborg, O. L., R. Miller and K. Ojima: Nutrient requirements of suspension cultures of soybean root cells. Exp. Cell Res., 50, 151158 (1968).

Giri, A. and M.L. Narasu: Transgenic hairy roots: Recent trends and applications. Biotechnol.Adv., 18, 1-22 (2000).

Guillon, S., J. Trémouillaux-Guiller, P.K. Pati, M. Rideau and P. Gantet: Harnessing the potential of hairy roots: Dawn of a new era. Trends Biotechnol., 24, 403-409 (2006)

Häkkinen, S.T., E. Moyano, R.M. Cusido and K.M. Oksman-Caldentey: Exploring the metabolic stability of engineered hairy roots after 16 years maintenance. Front. Plant Sci., 7, 1486 (2016)

Han Y.S., R. Van der Heijden and R. Verpoorte: Biosynthesis of anthraquinones in cell cultures of the Rubiaceae. Plant Cell Tissue Organ Cult., 67, 201-220 (2001)

Hanafya, M.S., M.A. Mattera, M.S. Askerb and M.R. Radya: Production of indole alkaloids in hairy root cultures of Catharanthus roseus $\mathrm{L}$. and their antimicrobial activity. Sou. Afri. J. Bot. 9, 9-18 (2016).

Hilton, M.G. and M.J.C. Rhodes: Growth and hyoscyamine production of 'hairy root'cultures of Datura stramonium in a modified stirred tank reactor. Appl. Microbiol. Biotechnol., 33, 132-138 (1990).

Huang, S.H., R.K. Vishwakarma, T.T. Lee, H.S. Chan and H.S. Tsay: Establishment of hairy root lines and analysis of iridoids and secoiridoids in the medicinal plant Gentiana scabra. Bot. Stud., 55, 17 (2014).

Jung, K.H., S.S Kwak, C.Y. Choi and J.R. Liu: Development of two stage culture process by optimization of inorganic salts for improving catharanthine production in hairy root cultures of Catharanthus roseus. J. Ferment. Bioeng., 77, 57-61 (1994).

Khare, N., D. Goyary, N.K. Singh, P. Shah, M. Rathore, S. Anandhan and Z. Ahmed: Transgenic tomato cv. PusaUphar expressing a bacterial mannitol-1-phosphate dehydrogenase gene confers abiotic stress tolerance. Plant Cell Tissue Organ Cult.,103, 267277 (2010).

Lang, B. and W.M. Kaiser: Solute content and energy status of roots of barley plants cultivated at different $\mathrm{pH}$ on nitrate- or ammoniumnitrogen. New Phytol., 128, 451-459 (1994).

Liu, W., R. Chen, M. Chen, H. Zhang, M. Peng, C. Yang, X. Ming, X. Lan and Z. Liao: Tryptophan decarboxylase plays an important role in ajmalicine biosynthesis in Rauvolûa verticillata. Planta, 236, 239-250 (2012).

Lorence, A., F. Medina-Bolivar and C.L. Nessler: Camptothecin and 10hydroxycamptothecin from Camptotheca acuminata hairy roots. Plant Cell Rep., 22, 437-441 (2004).

Lourenço, P.M., S. de Castro, T.M. Martins, A. Clemente and A. Domingos: Growth and proteolytic activity of hairy roots from Centaurea calcitrapa: Effect of nitrogen and sucrose. Enzyme Microb. Tech., 31, 242-249 (2002).

Malamy, J.E. and K.S. Ryan: Environmental regulation of lateral root initiation in Arabidopsis. Plant Physiol., 127, 899-909 (2001).
Murashige, T. and F. Skoog: A revised medium for rapid growth and bio assays with tobacco tissue cultures. Physiol. Plantarum, 15, 473497 (1962).

Mantell, S.H. and H. Smith: Cultural factors that influence secondary metabolite accumulations in plant cell and tissue cultures. In: Plant Biotechnology (Eds.: S.H. Mantell and H. Smith). Cambridge University Press, Cambridge, pp. 75-108 (1983).

Mehrotra, S., V. Srivastava, L.U. Rahman and A.K. Kukreja: Over expression of a Catharanthus tryptophan decarboxylase (tdc) gene leads to enhanced terpenoidindole alkaloid (TIA) production in transgenic hairy root lines of Rauwolfia serpentina. Plant Cell Tissue Organ Cult.,115, 377-384 (2013).

Murthy, H.N., C. Dijkstra, P. Anthony, D.A. White, M.R. Davey, J.B. Power, E.J. Hahn and Y.K. Paek: Establishment of Withania somnifera hairy root culturesfor the production of withanolide A. J. Int. Plant Biol., 50, 975-981 (2008).

Naik, P.M., S.H. Manohar, N. Praveenand and H.N. Murthy: Effects of sucrose and $\mathrm{pH}$ levels on in vitro shoot regeneration from leaf explants of Bacopa monnieri and accumulation of bacoside A in regenerated shoots. Plant Cell Tissue Organ Cult.,100, 235-239 (2010).

Nin, S., A. Bennici, G. Roselli, D. Mariotti, S. Schiff and R. Magherini: Agrobacterium-mediated transformation of Artemisia absinthium L. (wormwood) and production of secondary metabolites. Plant Cell Rep., 16, 725-730 (1997).

Nguyen, C., F. Bourgaud, P. Forlotand and A. Guckert: Establishment of hairy root cultures of Psoralea species. Plant Cell Rep., 11, 424427 (1992).

Pavlov, A., V. Georgiev and M. llieva: Betalain biosynthesis by red beet (Beta vulgaris L.) hairy root culture. Process Biochem., 40, 15311533 (2005).

Pathania, S., V. Randhawa and G. Bagler: Prospecting for novel plantderived molecules of Rauwolfia serpentina as inhibitors of aldose reductase, a potent drug target for diabetes and its complications. PlosONE, 8, e 61327 (2013).

Praveen, N. and H. N. Murthy: Withanolide A production from Withania somnifera hairy root cultures with improved growth by altering the concentrations of macro elements and nitrogen source in the medium. Acta Physiol. Plant., 35, 811-816 (2013).

Ray, S., A. Majumder, M. Bandyopadhyay and S. Jha: Genetic transformation of sarpagandha (Rauwolfia serpentina) with Agrobacterium rhizogenes for identification of high alkaloid yielding lines. Acta Physiol. Plant., 36, 1599-1605 (2014).

Sarma, D., A.K. Kukreja and A. Baruah: Transforming ability of two Agrobacterium rhizogenes strains in Rauwolfia serpentina (L.) leaves. Indian J. Plant Physiol., 2, 166-168 (1997).

Sandra, I., P. Alvarez and A.M. Giulietti: Influence of chitosan, acetic acid and citric acid on growth and tropane alkaloid production in transformed roots of Brugmansia candida: Effect of medium $\mathrm{pH}$ and growth phase. Plant Cell Tissue Organ Cult., 59, 31-38 (1999).

Sharafi, A., H.H. Sohi, A. Mousavi, P. Azadi, B.H. Khalifani and K. Razavi: Metabolic engineering of morphinane alkaloids by over expression of Codeinonereductase in transgenic hairy root of Papaver bracteatum. Biotechnol. Lett., 35, 445-453 (2013).

Shetty, M.R., G.A. Harisha, Y. Jayanth and A.H. Kumar: Production of secondary metabolites from in vitro cultures of Rauwolfia serpentina (L.) Benth. Int. J. Sci. Technol. Res., 2, 844-52 (2014).

Sivakumar, G., K.W. Yu, E.J. Hahn and K.Y. Paek: Optimization of 
organic nutrients for ginseng hairy roots production in large-scale bioreactors. Curr. Sci., 89, 641-649 (2005).

Srivastava, S. and A.K. Srivastava: Hairy root culture for massproduction of high-value secondary metabolites. Crit. Rev. Biotechnol., 27, 29-43 (2007).

Skała E., A. Kicel, M.A. Olszewska, A.K. Kiss and H. Wysokińska: Establishment of hairy root cultures of Rhaponticum carthamoides (Wild.) Iljin for the production of biomass and caffeic acid derivatives. Biomed. Res. Int., 2015, 181098 (2015).

Satdive R.K., D.P. Fulzele and S. Eapen: Studies on production of ajmalicine in shake flasks by multiple shoot cultures of Catharanthus roseus. Biotechnol. Prog., 19, 1071-1075(2003).

Taya, M., K. Mine, M. Kino-Oka, S. Tone and T. Ichi: Production and release of pigments by culture of transformed hairy root of red beet. J. Ferment. Bioeng., 73, 31-36 (1992).

Udomsuk, L., K. Jarukamjorn, H. Tanaka and W. Putalun: Isoflavonoid production in a hairy roots culture of Pueraria candollei. Z. Naturforsch. C., 64, 687-691 (2009).
Verma, V.C., H. Singh, A.S. Negi, G. Saxena, L. Rahmanand and S. Banerjee: Yield enhancement strategies for the production ofpicroliv from hairy root culture of Picrorhiza kurroa Royle ex Benth. Plant Signali. Behav., 10, e1023976 (2015).

Xu, H., J.H. Park, Y.K. Kim, N.I. Park, S.Y. Lee and S.U. Park: Optimization of growth and pyranocoumarins production in hairy root culture of Angelica gigas Nakai. J. Med. Plants Res., 3, 978981 (2009).

Young-Am, C., H.S. Yu, J.S. Song, H.K. Chun and S.U. Park: Indigo production in hairy root cultures of Polygonum tinctorium Lour. Biotechnol. Lett., 22, 1527-1530 (2000).

Yu, S., K.H. Kwok and P.M. Doran: Effect of sucrose, exogenous product concentration, and other culture conditions on growth and steroidal alkaloid production by Solanuma viculare hairy roots. Enzyme Microb. Technol., 18, 238-243 (1996).

Zhang, F.C., S.Z. Kang, F.S. Li and J.H. Zhang: Growth and major nutrient concentrations in Brassica campestris supplied with different $\mathrm{NH}_{4}{ }^{+} / \mathrm{NO}_{3}$ " ratios. J. Integr. Plant Biol., 49, 455-462 (2007). 